\title{
NEW SUBCLASS OF PSEUDO-TYPE MEROMORPHIC BI-UNIVALENT FUNCTIONS OF COMPLEX ORDER
}

\author{
G. Murugusundaramoorthy ${ }^{1, *}$, T. Janani, ${ }^{2}$, and K. Vijaya ${ }^{3}$ \\ $1, *$ Corresponding Author \\ School of Advanced Sciences, \\ VIT University, Vellore - 632 014, India. \\ E-mail : gmsmoorthy@yahoo.com \\ ${ }^{2}$ School of Computer Science and Engineering \\ VIT University, Vellore - 632 014, India. \\ E-mail: janani.t@vit.ac.in \\ ${ }^{3}$ School of Advanced Sciences, \\ VIT University, Vellore - 632 014, India. \\ E-mail :kvijaya@vit.ac.in
}

\begin{abstract}
In the present article, we define a new subclass of pseudo-type meromorphic biunivalent functions class $\Sigma^{\prime}$ of complex order $\gamma \in \mathbb{C} \backslash\{0\}$ and investigate the initial coefficient estimates $\left|b_{0}\right|,\left|b_{1}\right|$ and $\left|b_{2}\right|$. Further we mention several new or known consequences of our result.
\end{abstract}

AMS Subject Classification: 30C45, 30C50.

Keywords: Analytic functions; Univalent functions; Meromorphic functions; Bi-univalent functions of complex order; Coefficient bounds, Pseudo functions.

\section{IntRoduction AND Definitions}

Let $\mathcal{A}$ be class of analytic functions of the form

$$
f(z)=z+\sum_{n=2}^{\infty} a_{n} z^{n}
$$

which are univalent in the open unit disc

$$
\Delta=\{z:|z|<1\} .
$$

Also, let $\mathcal{S}$ be class of all functions in $\mathcal{A}$ which are univalent and normalized by the conditions

$$
f(0)=0=f^{\prime}(0)-1
$$

in $\Delta$. Some of the important and well-investigated subclasses of the univalent function class $\mathcal{S}$ includes the class $\mathcal{S}^{*}(\alpha)(0 \leq \alpha<1)$ of starlike functions of order $\alpha$ in $\Delta$ and the class $\mathcal{K}(\alpha)(0 \leq \alpha<1)$ of convex functions of order $\alpha$.

An analytic function $\varphi$ is subordinate to an analytic function $\psi$, written by $\varphi(z) \prec \psi(z)$, provided there is an analytic function $\omega$ defined on $\Delta$ with

$$
\omega(0)=0 \quad \text { and } \quad|\omega(z)|<1
$$

satisfying

$$
\varphi(z)=\psi(\omega(z))
$$


Ma and Minda [7] unified various subclasses of starlike and convex functions for which either of the quantity

$$
\frac{z f^{\prime}(z)}{f(z)} \text { or } 1+\frac{z f^{\prime \prime}(z)}{f^{\prime}(z)}
$$

is subordinate to a more general superordinate function. For this purpose, they considered an analytic function $\phi$ with positive real part in the unit disk $\Delta, \phi(0)=1, \phi^{\prime}(0)>0$ and $\phi$ maps $\Delta$ onto a region starlike with respect to 1 and symmetric with respect to the real axis.

The class of Ma-Minda starlike functions consists of functions $f \in \mathcal{A}$ satisfying the subordination

$$
\frac{z f^{\prime}(z)}{f(z)} \prec \phi(z)
$$

Similarly, the class of Ma-Minda convex functions consists of functions $f \in \mathcal{A}$ satisfying the subordination

$$
1+\frac{z f^{\prime \prime}(z)}{f^{\prime}(z)} \prec \phi(z)
$$

A function $f$ is bi-starlike of Ma-Minda type or bi-convex of Ma-Minda type if both $f$ and $f^{-1}$ are respectively starlike or convex. These classes are denoted respectively by $\mathcal{S}_{\Sigma}^{*}(\phi)$ and $\mathcal{K}_{\Sigma}(\phi)$. In the sequel, it is assumed that $\phi$ is an analytic function with positive real part in the unit disk $\Delta$, satisfying $\phi(0)=1, \phi^{\prime}(0)>0$ and $\phi(\Delta)$ is symmetric with respect to the real axis. Such a function has a series expansion of the form

$$
\phi(z)=1+B_{1} z+B_{2} z^{2}+B_{3} z^{3}+\cdots, \quad\left(B_{1}>0\right) .
$$

By setting $\phi(z)$ as given below:

$$
\phi(z)=\left(\frac{1+z}{1-z}\right)^{\alpha}=1+2 \alpha z+2 \alpha^{2} z^{2}+\frac{4 \alpha^{2}+2 \alpha}{3} z^{3}+\cdots \quad(0<\alpha \leq 1),
$$

we have

$$
B_{1}=2 \alpha, B_{2}=2 \alpha^{2} \text { and } \quad B_{3}=\frac{4 \alpha^{2}+2 \alpha}{3} .
$$

On the other hand, if we take

$$
\phi(z)=\frac{1+(1-2 \beta) z}{1-z}=1+2(1-\beta) z+2(1-\beta) z^{2}+\cdots \quad(0 \leq \beta<1),
$$

then

$$
B_{1}=B_{2}=B_{3}=2(1-\beta) .
$$

Let $\Sigma^{\prime}$ denote the class of meromorphic univalent functions $g$ of the form

$$
g(z)=z+b_{0}+\sum_{n=1}^{\infty} \frac{b_{n}}{z^{n}}
$$

defined on the domain $\Delta^{*}=\{z: 1<|z|<\infty\}$. Since $g \in \Sigma^{\prime}$ is univalent, it has an inverse $g^{-1}=h$ that satisfy

$$
g^{-1}(g(z))=z,\left(z \in \Delta^{*}\right)
$$

and

$$
g\left(g^{-1}(w)\right)=w,(M<|w|<\infty, M>0)
$$

where

$$
g^{-1}(w)=h(w)=w+\sum_{n=0}^{\infty} \frac{C_{n}}{w^{n}}, \quad(M<|w|<\infty) .
$$


Analogous to the bi-univalent analytic functions, a function $g \in \Sigma^{\prime}$ is said to be meromorphic bi-univalent if $g^{-1} \in \Sigma^{\prime}$. We denote the class of all meromorphic bi-univalent functions by $\mathcal{M}_{\Sigma^{\prime}}$. Estimates on the coefficients of meromorphic univalent functions were widely investigated in the literature, for example, Schiffer[11] obtained the estimate $\left|b_{2}\right| \leq \frac{2}{3}$ for meromorphic univalent functions $g \in \Sigma^{\prime}$ with $b_{0}=0$ and Duren [2] gave an elementary proof of the inequality $\left|b_{n}\right| \leq$ $\frac{2}{(n+1)}$ on the coefficient of meromorphic univalent functions $g \in \Sigma^{\prime}$ with $b_{k}=0$ for $1 \leq k<\frac{n}{2}$. For the coefficient of the inverse of meromorphic univalent functions $h \in \mathcal{M}_{\Sigma^{\prime}}$, Springer [12] proved that $\left|C_{3}\right| \leq 1 ;\left|C_{3}+\frac{1}{2} C_{1}^{2}\right| \leq \frac{1}{2}$ and conjectured that $\left|C_{2 n-1}\right| \leq \frac{(2 n-1) !}{n !(n-1) !}, \quad(n=1,2, \ldots)$.

In 1977, Kubota [6] has proved that the Springer's conjecture is true for $n=3,4,5$ and subsequently Schober [10] obtained a sharp bounds for the coefficients $C_{2 n-1}, 1 \leq n \leq 7$ of the inverse of meromorphic univalent functions in $\Delta^{*}$. Recently, Kapoor and Mishra [5] (see [13]) found the coefficient estimates for a class consisting of inverses of meromorphic starlike univalent functions of order $\alpha$ in $\Delta^{*}$.

Recently, Babalola [1] defined a new subclass $\lambda$-pseudo starlike function of order $\beta(0 \leq \beta<$ 1) satisfying the analytic condition

$$
\Re\left(\frac{z\left(f^{\prime}(z)\right)^{\lambda}}{f(z)}\right)>\beta, \quad(z \in \mathbb{U}, \lambda \geq 1 \in \mathbb{R})
$$

and denoted by $\mathcal{L}_{\lambda}(\beta)$. Babalola 1 remarked that though for $\lambda>1$, these classes of $\lambda$-pseudo starlike functions clone the analytic representation of starlike functions. Also, when $\lambda=1$, we have the class of starlike functions of order $\beta(1-$ pseudo starlike functions of order $\beta)$ and for $\lambda=2$, we have the class of functions, which is a product combination of geometric expressions for bounded turning and starlike functions.

Motivated by the earlier work of [3, 4, 15, 8, 14, 15], in the present investigation, we define a new subclass of pseudo type meromorphic bi-univalent functions class $\Sigma^{\prime}$ of complex order $\gamma \in \mathbb{C} \backslash\{0\}$, and the estimates for the coefficients $\left|b_{0}\right|,\left|b_{1}\right|$ and $\left|b_{2}\right|$ are investigated. Several new consequences of the results are also pointed out.

Definition 1.1. For $0<\lambda \leq 1$ and $\mu \geq 1$, a function $g(z) \in \Sigma^{\prime}$ given by (1.5) is said to be in the class $\mathcal{P}_{\Sigma^{\prime}}^{\gamma}(\lambda, \mu, \phi)$ if the following conditions are satisfied:

$$
1+\frac{1}{\gamma}\left[(1-\lambda)\left(\frac{g(z)}{z}\right)^{\mu}+\lambda\left(\frac{z\left(g^{\prime}(z)\right)^{\mu}}{g(z)}\right)-1\right] \prec \phi(z)
$$

and

$$
1+\frac{1}{\gamma}\left[(1-\lambda)\left(\frac{h(w)}{w}\right)^{\mu}+\lambda\left(\frac{w\left(h^{\prime}(w)\right)^{\mu}}{h(w)}\right)-1\right] \prec \phi(w)
$$

where $z, w \in \Delta^{*}, \gamma \in \mathbb{C} \backslash\{0\}$ and the function $h$ is given by (1.6).

By suitably specializing the parameter $\lambda$, we state new subclass of meromorphic pseudo bi-univalent functions of complex order $\mathcal{P}_{\Sigma^{\prime}}^{\gamma}(\lambda, \mu, \phi)$ as illustrated in the following Examples.

Example 1.1. For $\lambda=1$, a function $g \in \Sigma^{\prime}$ given by (1.5) is said to be in the class $\mathcal{P}_{\Sigma^{\prime}}^{\gamma}(1, \mu, \phi) \equiv \mathcal{P}_{\Sigma^{\prime}}^{\gamma}(\mu, \phi)$ if it satisfies the following conditions:

$$
1+\frac{1}{\gamma}\left(\frac{z\left(g^{\prime}(z)\right)^{\mu}}{g(z)}-1\right) \prec \phi(z) \quad \text { and } \quad 1+\frac{1}{\gamma}\left(\frac{w\left(h^{\prime}(w)\right)^{\mu}}{h(w)}-1\right) \prec \phi(w)
$$

where $z, w \in \Delta^{*}, \mu \geq 1, \gamma \in \mathbb{C} \backslash\{0\}$ and the function $h$ is given by (1.6). 
Example 1.2. For $\lambda=1$ and $\gamma=1$, a function $g \in \Sigma^{\prime}$ given by (1.5) is said to be in the class $\mathcal{P}_{\Sigma^{\prime}}^{1}(1, \mu, \phi) \equiv \mathcal{P}_{\Sigma^{\prime}}(\mu, \phi)$ if it satisfies the following conditions :

$$
\frac{z\left(g^{\prime}(z)\right)^{\mu}}{g(z)} \prec \phi(z) \quad \text { and } \quad \frac{w\left(h^{\prime}(w)\right)^{\mu}}{h(w)} \prec \phi(w)
$$

where $z, w \in \Delta^{*}, \mu \geq 1$ and the function $h$ is given by (1.6).

\section{Coefficient estimates for NeW function CLASs $\mathcal{P}_{\Sigma^{\prime}}^{\gamma}(\lambda, \mu, \phi)$}

In this section, we obtain the coefficient estimates $\left|b_{0}\right|,\left|b_{1}\right|$ and $\left|b_{2}\right|$ for $\mathcal{P}_{\Sigma^{\prime}}^{\gamma}(\lambda, \mu, \phi)$, a new subclass of meromorphic pseudo bi-univalent functions class $\Sigma^{\prime}$ of complex order $\gamma \in \mathbb{C} \backslash\{0\}$. In order to prove our result, we recall the following lemma.

Lemma 2.1. [9] If $\Phi \in \mathcal{P}$, the class of all functions with $\Re(\Phi(z))>0,(z \in \Delta)$ then

$$
\left|c_{k}\right| \leq 2 \text {, for each } k \text {, }
$$

where

$$
\Phi(z)=1+c_{1} z+c_{2} z^{2}+\cdots \text { for } z \in \Delta .
$$

Define the functions $p$ and $q$ in $\mathcal{P}$ given by

$$
p(z)=\frac{1+u(z)}{1-u(z)}=1+\frac{p_{1}}{z}+\frac{p_{2}}{z^{2}}+\cdots
$$

and

$$
q(z)=\frac{1+v(z)}{1-v(z)}=1+\frac{q_{1}}{z}+\frac{q_{2}}{z^{2}}+\cdots
$$

It follows that

$$
u(z)=\frac{p(z)-1}{p(z)+1}=\frac{1}{2}\left[\frac{p_{1}}{z}+\left(p_{2}-\frac{p_{1}^{2}}{2}\right) \frac{1}{z^{2}}+\cdots\right]
$$

and

$$
v(z)=\frac{q(z)-1}{q(z)+1}=\frac{1}{2}\left[\frac{q_{1}}{z}+\left(q_{2}-\frac{q_{1}^{2}}{2}\right) \frac{1}{z^{2}}+\cdots\right] .
$$

Note that for the functions $p(z), q(z) \in \mathcal{P}$, we have

$$
\left|p_{i}\right| \leq 2 \text { and }\left|q_{i}\right| \leq 2 \text { for each } i \text {. }
$$

Theorem 2.1. Let $g$ be given by (1.5) in the class $\mathcal{P}_{\Sigma^{\prime}}^{\gamma}(\lambda, \mu, \phi)$. Then

$$
\begin{gathered}
\left|b_{0}\right| \leq \frac{|\gamma|\left|B_{1}\right|}{|\mu-\mu \lambda-\lambda|}, \\
\left|b_{1}\right| \leq \frac{|\gamma|}{2|\mu-\lambda-2 \mu \lambda|} \sqrt{4\left|\left(B_{1}-B_{2}\right)^{2}\right|+4\left|B_{1}^{2}\right|+8\left|B_{1}\left(B_{1}-B_{2}\right)\right|+\frac{|\mu(\mu-1)(1-\lambda)+2 \lambda|^{2}\left|\gamma^{2} B_{1}^{4}\right|}{|\mu-\mu \lambda-\lambda|^{4}}}
\end{gathered}
$$

and

$\left|b_{2}\right| \leq \frac{|\gamma|}{2|\mu-\lambda-3 \mu \lambda|}\left(2\left|B_{1}\right|+4\left|B_{2}-B_{1}\right|+2\left|B_{1}-2 B_{2}+B_{3}\right|+\frac{|\mu(\mu-1)(\mu-2)(1-\lambda)-6 \lambda||\gamma|^{2}\left|B_{1}\right|^{3}}{3|\lambda|^{3}}\right)$

where $\gamma \in \mathbb{C} \backslash\{0\}, 0<\lambda \leq 1, \mu \geq 1$ and $z, w \in \Delta^{*}$. 
Proof. It follows from (1.8) and (1.9) that

$$
1+\frac{1}{\gamma}\left[(1-\lambda)\left(\frac{g(z)}{z}\right)^{\mu}+\lambda\left(\frac{z\left(g^{\prime}(z)\right)^{\mu}}{g(z)}\right)-1\right]=\phi(u(z))
$$

and

$$
1+\frac{1}{\gamma}\left[(1-\lambda)\left(\frac{h(w)}{w}\right)^{\mu}+\lambda\left(\frac{w\left(h^{\prime}(w)\right)^{\mu}}{h(w)}\right)-1\right]=\phi(v(w)) .
$$

In light of (1.5), (1.6), (1.8) and (1.9), we have

$$
\begin{aligned}
1+\frac{1}{\gamma}\left[(1-\lambda)\left(\frac{g(z)}{z}\right)^{\mu}+\lambda\left(\frac{z\left(g^{\prime}(z)\right)^{\mu}}{g(z)}\right)-1\right] & \\
=1+ & B_{1} p_{1} \frac{1}{2 z}+\left[\frac{1}{2} B_{1}\left(p_{2}-\frac{p_{1}^{2}}{2}\right)+\frac{1}{4} B_{2} p_{1}^{2}\right] \frac{1}{z^{2}} \\
+ & {\left[\frac{B_{1}}{2}\left(p_{3}-p_{1} p_{2}+\frac{p_{1}^{3}}{4}\right)+\frac{B_{2}}{2}\left(p_{1} p_{2}-\frac{p_{1}^{3}}{2}\right)+B_{3} \frac{p_{1}^{3}}{8}\right] \frac{1}{z^{3}} \ldots }
\end{aligned}
$$

and

$$
\begin{aligned}
1+\frac{1}{\gamma}\left[(1-\lambda)\left(\frac{h(w)}{w}\right)^{\mu}+\right. & \left.\lambda\left(\frac{w\left(h^{\prime}(w)\right)^{\mu}}{h(w)}\right)-1\right] \\
=1+ & B_{1} q_{1} \frac{1}{2 w}+\left[\frac{1}{2} B_{1}\left(q_{2}-\frac{q_{1}^{2}}{2}\right)+\frac{1}{4} B_{2} q_{1}^{2}\right] \frac{1}{w^{2}} \\
+ & {\left[\frac{B_{1}}{2}\left(q_{3}-q_{1} q_{2}+\frac{q_{1}^{3}}{4}\right)+\frac{B_{2}}{2}\left(q_{1} q_{2}-\frac{q_{1}^{3}}{2}\right)+B_{3} \frac{q_{1}^{3}}{8}\right] \frac{1}{w^{3}} \ldots }
\end{aligned}
$$

Now, equating the coefficients in (2.6) and (2.7), we get

$$
\begin{gathered}
\frac{(\mu-\mu \lambda-\lambda)}{\gamma} b_{0}=\frac{1}{2} B_{1} p_{1} \\
\frac{1}{2 \gamma}\left[(\mu(\mu-1)(1-\lambda)+2 \lambda) b_{0}^{2}+2(\mu-\lambda-2 \lambda \mu) b_{1}\right]=\frac{1}{2} B_{1}\left(p_{2}-\frac{p_{1}^{2}}{2}\right)+\frac{1}{4} B_{2} p_{1}^{2}, \\
\frac{1}{6 \gamma}\left[(\mu(\mu-1)(\mu-2)(1-\lambda)-6 \lambda) b_{0}^{3}+6(\mu(\mu-1)(1-\lambda)+2 \lambda+\lambda \mu) b_{0} b_{1}+6(\mu-\lambda-3 \lambda \mu) b_{2}\right] \\
=\left[\frac{B_{1}}{2}\left(p_{3}-p_{1} p_{2}+\frac{p_{1}^{3}}{4}\right)+\frac{B_{2}}{2}\left(p_{1} p_{2}-\frac{p_{1}^{3}}{2}\right)+B_{3} \frac{p_{1}^{3}}{8}\right] \\
\frac{-(\mu-\mu \lambda-\lambda)}{\gamma} b_{0}=\frac{1}{2} B_{1} q_{1} \\
\frac{1}{2 \gamma}\left[(\mu(\mu-1)(1-\lambda)+2 \lambda) b_{0}^{2}+2(\lambda-\mu+2 \lambda \mu) b_{1}\right]=\frac{1}{2} B_{1}\left(q_{2}-\frac{q_{1}^{2}}{2}\right)+\frac{1}{4} B_{2} q_{1}^{2}
\end{gathered}
$$

and

$$
\begin{gathered}
\frac{1}{6 \gamma}\left[(6 \lambda-\mu(\mu-1)(\mu-2)(1-\lambda)) b_{0}^{3}+6(\mu(\mu-1)(1-\lambda)-\mu(1-\lambda)+3 \lambda+3 \lambda \mu) b_{0} b_{1}+6(\lambda-\mu+3 \lambda \mu) b_{2}\right] \\
=\left[\frac{B_{1}}{2}\left(q_{3}-q_{1} q_{2}+\frac{q_{1}^{3}}{4}\right)+\frac{B_{2}}{2}\left(q_{1} q_{2}-\frac{q_{1}^{3}}{2}\right)+B_{3} \frac{q_{1}^{3}}{8}\right]
\end{gathered}
$$


From (2.8) and (2.11), we get

$$
p_{1}=-q_{1}
$$

and

$$
b_{0}^{2}=\frac{\gamma^{2} B_{1}^{2}}{8(\mu-\mu \lambda-\lambda)^{2}}\left(p_{1}^{2}+q_{1}^{2}\right) .
$$

Applying Lemma (2.1) for the coefficients $p_{1}$ and $q_{1}$, we have

$$
\left|b_{0}\right| \leq \frac{|\gamma|\left|B_{1}\right|}{|\mu-\mu \lambda-\lambda|}
$$

Next, in order to find the bound on $\left|b_{1}\right|$ from (2.9), (2.12) (2.14) and(2.15), we obtain

$$
\begin{aligned}
2(\mu-\lambda-2 \lambda \mu)^{2} & \frac{b_{1}^{2}}{\gamma^{2}}+[\mu(\mu-1)(1-\lambda)+2 \lambda]^{2} \frac{b_{0}^{4}}{2 \gamma^{2}} \\
& =\left(B_{1}-B_{2}\right)^{2} \frac{p_{1}^{4}}{8}+\frac{B_{1}^{2}}{4}\left(p_{2}^{2}+q_{2}^{2}\right)+B_{1}\left(B_{2}-B_{1}\right) \frac{\left(p_{1}^{2} p_{2}+q_{1}^{2} q_{2}\right)}{4} .
\end{aligned}
$$

Using (2.15) and applying Lemma (2.1) once again for the coefficients $p_{1}, p_{2}$ and $q_{2}$, we get

$$
\begin{aligned}
\left|b_{1}\right|^{2} \leq & \frac{\left|\gamma^{2}\right|}{4|\mu-\lambda-2 \lambda \mu|^{2}} \times \\
& \quad\left(4\left|\left(B_{1}-B_{2}\right)^{2}\right|+4\left|B_{1}\right|^{2}+8\left|B_{1}\left(B_{1}-B_{2}\right)\right|+\frac{|\mu(\mu-1)(1-\lambda)+2 \lambda|^{2}\left|\gamma^{2} B_{1}^{4}\right|}{|\mu-\mu \lambda-\lambda|^{4}}\right) .
\end{aligned}
$$

That is,

$$
\begin{aligned}
& \left|b_{1}\right| \leq \frac{|\gamma|}{2|\mu-\lambda-2 \lambda \mu|} \times \\
& \qquad \sqrt{4\left|\left(B_{1}-B_{2}\right)^{2}\right|+4\left|B_{1}\right|^{2}+8\left|B_{1}\left(B_{1}-B_{2}\right)\right|+\frac{|\mu(\mu-1)(1-\lambda)+2 \lambda|^{2}\left|\gamma^{2} B_{1}^{4}\right|}{|\mu-\mu \lambda-\lambda|^{4}}} .
\end{aligned}
$$

In order to find the estimate $\left|b_{2}\right|$, consider the sum of (2.10) and (2.13) with $p_{1}=-q_{1}$, we have

$$
\frac{1}{\gamma} b_{0} b_{1}=\frac{B_{1}\left[p_{3}+q_{3}\right]+\left(B_{2}-B_{1}\right) p_{1}\left[p_{2}-q_{2}\right]}{2[2 \mu(\mu-1)(1-\lambda)-(1-\lambda) \mu+5 \lambda+4 \lambda \mu]} .
$$

Subtracting (2.13) from (2.10) and using $p_{1}=-q_{1}$ we have

$$
\begin{aligned}
& 2(\mu-\lambda-3 \lambda \mu) \frac{b_{2}}{\gamma} \\
&=-(\mu-\lambda-3 \mu \lambda) \frac{b_{0} b_{1}}{\gamma}-[\mu(\mu-1)(\mu-2)(1-\lambda)-6 \lambda] \frac{b_{0}^{3}}{3 \gamma}+\frac{B_{1}}{2}\left(p_{3}-q_{3}\right) \\
&+\frac{B_{2}-B_{1}}{2}\left(p_{2}+q_{2}\right) p_{1}+\frac{B_{1}-2 B_{2}+B_{3}}{4} p_{1}^{3} .
\end{aligned}
$$


Substituting for $\frac{b_{0} b_{1}}{\gamma}$ and $\frac{b_{0}^{3}}{\gamma}$ in (2.18) , simple computation yields,

$$
\begin{aligned}
\frac{b_{2}}{\gamma} & =\frac{-B_{1}}{2(\mu-\lambda-3 \lambda \mu)}\left(\frac{\mu-3 \lambda-4 \lambda \mu-\mu(\mu-1)(1-\lambda)}{2 \mu(\mu-1)(1-\lambda)-\mu+5 \lambda+5 \lambda \mu} p_{3}+\frac{2 \lambda+\lambda \mu+\mu(\mu-1)(1-\lambda)}{2 \mu(\mu-1)(1-\lambda)-\mu+5 \lambda+5 \lambda \mu} q_{3}\right) \\
& -\frac{\left(B_{2}-B_{1}\right) p_{1}}{2(\mu-\lambda-3 \lambda \mu)}\left(\frac{\mu-3 \lambda-4 \lambda \mu-\mu(\mu-1)(1-\lambda)}{2 \mu(\mu-1)(1-\lambda)-\mu+5 \lambda+5 \lambda \mu} p_{2}-\frac{2 \lambda+\lambda \mu+\mu(\mu-1)(1-\lambda)}{2 \mu(\mu-1)(1-\lambda)-\mu+5 \lambda+5 \lambda \mu} q_{2}\right) \\
& +\frac{B_{1}-2 B_{2}+B_{3}}{8(\mu-\lambda-3 \lambda \mu)} p_{1}^{3}-\frac{(\mu(\mu-1)(\mu-2)(1-\lambda)-6 \lambda) \gamma^{2} B_{1}^{3}}{48(\mu-\lambda-3 \lambda \mu) \lambda^{3}} p_{1}^{3} .
\end{aligned}
$$

Applying Lemma 2.1 in the above equation yields,

$$
\begin{aligned}
\left|b_{2}\right| \leq & \frac{|\gamma|}{2|\mu-\lambda-3 \lambda \mu|} \times \\
& \left(2\left|B_{1}\right|+4\left|B_{2}-B_{1}\right|+2\left|B_{1}-2 B_{2}+B_{3}\right|+\frac{|\mu(\mu-1)(\mu-2)(1-\lambda)-6 \lambda||\gamma|^{2}\left|B_{1}\right|^{3}}{3|\lambda|^{3}}\right)
\end{aligned}
$$

By taking $\lambda=1$, we state the following.

Theorem 2.2. Let $g$ be given by (1.5) in the class $\mathcal{P}_{\Sigma^{\prime}}^{\gamma}(\mu, \phi)$. Then

$$
\begin{gathered}
\left|b_{0}\right| \leq|\gamma|\left|B_{1}\right| \\
\left|b_{1}\right| \leq \frac{|\gamma|}{|1+\mu|} \sqrt{\left|\left(B_{1}-B_{2}\right)^{2}\right|+\left|B_{1}^{2}\right|+2\left|B_{1}\left(B_{1}-B_{2}\right)\right|+|\gamma|^{2}\left|B_{1}^{4}\right|} .
\end{gathered}
$$

and

$$
\left|b_{2}\right| \leq \frac{|\gamma|}{|1+2 \mu|}\left(\left|B_{1}\right|+2\left|B_{2}-B_{1}\right|+\left|B_{1}-2 B_{2}+B_{3}\right|+|\gamma|^{2}\left|B_{1}\right|^{3}\right)
$$

where $\gamma \in \mathbb{C} \backslash\{0\}, \mu \geq 1$ and $z, w \in \Delta^{*}$.

By taking $\lambda=1$ and $\gamma=1$, we state the following results.

Theorem 2.3. Let $g$ be given by (1.5) in the class $\mathcal{P}_{\Sigma^{\prime}}(\mu, \phi)$. Then

$$
\left|b_{1}\right| \leq \frac{1}{|1+\mu|} \sqrt{\left|\left(B_{1}-B_{2}\right)^{2}\right|+\left|B_{1}^{2}\right|+2\left|B_{1}\left(B_{1}-B_{2}\right)\right|+\left|B_{1}^{4}\right|} .
$$

and

$$
\left|b_{2}\right| \leq \frac{1}{|1+2 \mu|}\left(\left|B_{1}\right|+2\left|B_{2}-B_{1}\right|+\left|B_{1}-2 B_{2}+B_{3}\right|+\left|B_{1}\right|^{3}\right)
$$

where $\mu \geq 1, z, w \in \Delta^{*}$.

\section{Corollaries and concluding Remarks}

Corollary 3.1. Let $g$ be given by (1.5) in the class $\mathcal{P}_{\Sigma^{\prime}}^{\gamma}\left(\lambda, \mu,\left(\frac{1+z}{1-z}\right)^{\alpha}\right) \equiv \mathcal{P}_{\Sigma^{\prime}}^{\gamma}(\lambda, \mu, \alpha)$. Then

$$
\begin{gathered}
\left|b_{0}\right| \leq \frac{2|\gamma| \alpha}{|\mu-\mu \lambda-\lambda|}, \\
\left|b_{1}\right| \leq \frac{2|\gamma| \alpha}{|\mu-\lambda-2 \lambda \mu|} \sqrt{(\alpha-2)^{2}+\frac{|\mu(\mu-1)(1-\lambda)+2 \lambda|^{2}\left|\gamma^{2}\right|}{|\mu-\mu \lambda-\lambda|^{4}} \alpha^{2}}
\end{gathered}
$$


and

$$
\left|b_{2}\right| \leq \frac{2|\gamma| \alpha}{|\mu-\lambda-3 \lambda \mu|}\left(3-2 \alpha+\left(\frac{4-6 \alpha+2 \alpha^{2}}{3}\right)+\frac{2|\gamma|^{2} \alpha^{2}|\mu(\mu-1)(\mu-2)(1-\lambda)-6 \lambda|}{3|\lambda|^{3}}\right)
$$

where $\gamma \in \mathbb{C} \backslash\{0\}, 0<\lambda \leq 1, \mu \geq 1$ and $z, w \in \Delta^{*}$.

Corollary 3.2. Let $g$ be given by (1.5) in the class $\mathcal{P}_{\Sigma^{\prime}}^{\gamma}\left(\lambda, \mu, \frac{1+(1-2 \beta) z}{1-z}\right) \equiv \mathcal{P}_{\Sigma^{\prime}}^{\gamma}(\lambda, \mu, \beta)$. Then

$$
\begin{gathered}
\left|b_{0}\right| \leq \frac{2|\gamma|(1-\beta)}{|\mu-\mu \lambda-\lambda|}, \\
\left|b_{1}\right| \leq \frac{2|\gamma|(1-\beta)}{|\mu-\lambda-2 \lambda \mu|} \sqrt{1+\frac{|\mu(\mu-1)(1-\lambda)+2 \lambda|^{2}\left|\gamma^{2}\right|}{|\mu-\mu \lambda-\lambda|^{4}}(1-\beta)^{2}}
\end{gathered}
$$

and

$$
\left|b_{2}\right| \leq \frac{2|\gamma|(1-\beta)}{|\mu-\lambda-3 \lambda \mu|}\left(1+\frac{2|\gamma|^{2}(1-\beta)^{2}|\mu(\mu-1)(\mu-2)(1-\lambda)-6 \lambda|}{3|\lambda|^{3}}\right)
$$

where $\gamma \in \mathbb{C} \backslash\{0\}, 0<\lambda \leq 1, \mu \geq 1$ and $z, w \in \Delta^{*}$.

Concluding Remarks: We remark that, when $\lambda=1$ and $\mu=1$, the class $\mathcal{P}_{\Sigma^{\prime}}^{\gamma}(\lambda, \mu, \phi) \equiv$ $\mathcal{S}_{\Sigma^{\prime}}^{\gamma}(\phi)$, the subclass of meromorphic bi-starlike functions of complex order. By taking $\mu=1$ in the Theorem 2.2, we can easily obtain the coefficient estimates $b_{0}, b_{1}$ and $b_{2}$ for $\mathcal{S}_{\Sigma^{\prime}}^{\gamma}(\phi)$, which leads to the results discussed in Theorem 2.3 of [8]. Also, we can obtain the initial coefficient estimates for function $g$ given by (1.5) in the subclass $\mathcal{S}_{\Sigma^{\prime}}^{\gamma}(\phi)$ by taking $\phi(z)$ given in (1.3) and (1.4) respectively.

Future Work: Let a function $g \in \Sigma^{\prime}$ be given by (1.5). By taking $\gamma=(1-\alpha) \cos \beta e^{-i \beta},|\beta|<$ $\frac{\pi}{2}, 0 \leq \alpha<1$, the class $\mathcal{P}_{\Sigma^{\prime}}^{\gamma}(\lambda, \mu, \phi) \equiv \mathcal{P}_{\Sigma^{\prime}}^{\beta}(\alpha, \lambda, \mu, \phi)$ called the generalized class of $\beta$ bi-spiral like functions of order $\alpha(0 \leq \alpha<1)$ satisfying the following conditions.

$$
e^{i \beta}\left[(1-\lambda)\left(\frac{g(z)}{z}\right)^{\mu}+\lambda\left(\frac{z\left(g^{\prime}(z)\right)^{\mu}}{g(z)}\right)-1\right] \prec[\phi(z)(1-\alpha)+\alpha] \cos \beta+i \sin \beta
$$

and

$$
e^{i \beta}\left[(1-\lambda)\left(\frac{h(w)}{w}\right)^{\mu}+\lambda\left(\frac{w\left(h^{\prime}(w)\right)^{\mu}}{h(w)}\right)-1\right] \prec[\phi(w)(1-\alpha)+\alpha] \cos \beta+i \sin \beta
$$

where $0<\lambda \leq 1, \mu \geq 1, z, w \in \Delta^{*}$ and the function $h$ is given by (1.6).

For function $g \in \mathcal{P}_{\Sigma^{\prime}}^{\beta}(\alpha, \lambda, \mu, \phi)$ given by (1.5), by choosing $\phi(z)=\left(\frac{1+z}{1-z}\right)$, (or $\phi(z)=$ $\left.\frac{1+A z}{1+B z},-1 \leq B<A \leq 1\right)$, we can obtain the estimates $\left|b_{0}\right|,\left|b_{1}\right|$ and $\left|b_{2}\right|$ by routine procedure (as in Theorem 2.1) and so we omit the details.

\section{REFERENCES}

[1] K. O. Babalola, On $\lambda$-pseudo starlike functions, Journal of Classical Analysis, 3(2)(2013), 137 - 147.

[2] P. L. Duren, Coefficients of meromorphic schlicht functions, Proceedings of the American Mathematical Society, 28(1971), 169-172.

[3] E. Deniz, Certain subclasses of bi-univalent functions satisfying subordinate conditions, Journal of Classical Analysis, 2(1) (2013), 49-60.

[4] T. Janani and G. Murugusundaramoorthy, Coefficient estimates of meromorphic bi- starlike functions of complex order, International Journal of Analysis and Applications, 4(1)(2014), 68-77.

[5] G. P. Kapoor and A. K. Mishra, Coefficient estimates for inverses of starlike functions of positive order, Journal of Mathematical Analysis and Applications, 329(2)( 2007), 922-934. 
[6] Y. Kubota, Coefficients of meromorphic univalent functions, Kodai Mathematical Seminar Reports, 28(2-3) (1977), 253-261.

[7] W.C. Ma, D. Minda, A unified treatment of some special classes of functions, in: Proceedings of the Conference on Complex Analysis, Tianjin, 1992, 157 - 169, Conf. Proc.Lecture Notes Anal. 1. Int. Press, Cambridge, MA, 1994.

[8] G. Murugusundaramoorthy, T. Janani and NE. Cho, Coefficient estimates of Mocanu type meromorphic bi-univalent functions of complex order, Proceedings of the Jangjeon Mathematical Society, 19(2016), 691700.

[9] C. Pommerenke, Univalent Functions, Vandenhoeck \& Ruprecht, Göttingen, 1975.

[10] G. Schober, Coefficients of inverses of meromorphic univalent functions, Proceedings of the American Mathematical Society, 67(1)(1977), 111-116.

[11] M. Schiffer, On an extremum problem of conformal representation, Bulletin de la Socit Mathmatique de France, 66 (1938), 48-55.

[12] G. Springer, The coefficient problem for schlicht mappings of the exterior of the unit circle, Transactions of the American Mathematical Society, 70 (1951), 421-450.

[13] H. M. Srivastava, A. K. Mishra, and S. N. Kund, Coefficient estimates for the inverses of starlike functions represented by symmetric gap series, Panamerican Mathematical Journal, 21(4)(2011), 105-123.

[14] H. M. Srivastava, Santosh B. Joshi, Sayali S. Joshi and Haridas Pawar, Coefficient Estimates for Certain Subclasses of Meromorphically Bi-Univalent Functions, Palestine Journal of Mathematics, 5 (Special Issue: 1) (2016), 250-258.

[15] Q-H. Xu , Chun-Bo Lv , H.M. Srivastava Coefficient estimates for the inverses of a certain general class of spirallike functions, Applied Mathematics and Computation, 219 (2013), 7000-7011. 